\title{
Comparación de resultados visuales y de calidad de visión después del implante bilateral de lentes intraoculares trifocales frente a lentes intraoculares bifocales
}

\section{Comparison of visual results and quality of vision after bilateral implantation of trifocal intraocular lenses vs bifocal intraocular lenses}

\author{
Diego Zamora-de-la-Cruz*, Marisol Garzón y Eduardo Chávez-Mondragón \\ Departamento de Segmento Anterior, Instituto de Oftalmología Fundación Conde de Valenciana I.A.P., Ciudad de México, México
}

\begin{abstract}
Resumen
Objetivo: Determinar y comparar los resultados visuales entre el implante bilateral de lente intraocular (LIO) bifocal ReSTOR +2.50 y LIO trifocal PanOptix en pacientes con catarata y presbicia en el Instituto de Oftalmología Fundación Conde de Valenciana en Ciudad de México. Material y métodos: Se evaluaron 24 ojos de 12 pacientes con implante de ReSTOR +2.50 y PanOptix en el Instituto de Oftalmología Fundación Conde de Valenciana en un periodo de seis meses. Se evaluaron la agudeza visual mejor corregida, a diferentes distancias (de lejos, intermedia y de cerca), y la sensibilidad al contraste en condiciones fotópicas a diferentes frecuencias espaciales. Se realizó una curva de desenfoque monocular y se evaluó el cuestionario VFQ-25. Resultados: Las agudezas visuales de lejos fueron buenas en ambos grupos, aunque las diferencias de agudezas visuales intermedias y de cerca fueron clínica y estadísticamente significativas entre ambos LIO, diferencias que favorecieron a lio PanOptix. Las pruebas de sensibilidad al contraste no mostraron diferencias notables entre ambos lentes. Los resultados de la encuesta VFQ-25 para ambos lentes fueron altos y no mostraron diferencias importantes entre sí. Conclusiones: Ambos lentes muestran tener buena aceptación entre los pacientes. Parece ser que, actualmente, el único criterio aceptable para decidir entre un lente u otro se basa básicamente en las necesidades de visión del paciente, que están íntimamente relacionadas con la edad y las actividades que éste realice.
\end{abstract}

Palabras clave: Lentes intraoculares. Presbicia. Facoemulsificación. Sensibilidad al contraste.

\section{Abstract}

Purpose: To evaluate and compare visual outcomes of visual quality between bilateral implantation of a bifocal intraocular lens (IOL) (ReSTOR +2.50) and a trifocal IOL (PanOptix) in patients with cataract and presbyopia at Ophthalmology Institute Fundación Conde de Valenciana in Mexico City. Materials and Methods: We evaluated 24 eyes of 12 patients with ReSTOR +2.50 and PanOptix at the Ophtalmology Institute "Fundación Conde de Valenciana" in a period of 6 months. The best corrected visual acuity at different distances (distant, intermediate and near) and contrast sensitivity in photopic conditions at 
different frequencies were evaluated. A binocular defocus curve was obtained, and subjective visual function and quality of vision were evaluated by the VFQ-25 questionaire. Results: The far visual acuities between the two groups were acceptable in both groups, the differences in intermediate and near visual acuity were clinically and statistically significant between both IOL, these differences favored PanOptix IOL. Contrast-sensitivity values did not show noticeable differences between the two groups. VFQ-25 questionaire results for both lenses were high and showed no significant differences between them. Conclusions: Both lenses show good acceptance among patients, it seems that currently the only acceptable criteria for deciding between both lenses are patient's vision needs and are closely related to age and current activities.

KEY WORDS: Intraocular lenses. Presbyopia. Phacoemulsification. Contrast sensitivity.

\section{Introducción}

La catarata es, hoy en día, la principal causa de ceguera reversible en el mundo y en nuestro país ${ }^{1}$. La cirugía de catarata, específicamente la facoemulsificación, es la que más se realiza en todo el mundo. Con el avance en la tecnología ha crecido el desarrollo de nuevos LIO, con los cuales se pretende restaurar la función original del cristalino para enfocar objetos a diferentes distancias: de lejos, intermedia y de cerca.

Los LIO multifocales se basan en una óptica difractiva para lograr diferentes rangos de visión (de cerca, intermedia y de lejos), lo cual significa que dividen la luz en varios puntos focales: uno para visión de cerca, otro para visión intermedia y otro para visión de lejos, con la finalidad de corregir la presbicia que se asocia a la pérdida de la capacidad de acomodación del cristalino con la edad ${ }^{2-4}$.

Actualmente se han desarrollado diseños difractivos en LIO, como el ReSTOR +2.50 (Alcon, Forthworth TX, EE.UU.). Éste y otros LIO multifocales buscan desarrollar perfiles asféricos con adición baja y simetría no rotacional para lograr mejores rangos de visión, entre los que se encuentra también la visión intermedia $(60-80 \mathrm{~cm})^{4-6}$.

El lente ReSTOR +2.50 (SV25T0) es un LIO multifocal y bifocal indicado para pacientes que tienen necesidad de trabajar a distancias intermedias, sobre todo a 40-70 cm de distancia. Tiene anillos concéntricos en la parte central de la óptica y otorga a los pacientes una adición en la parte central de +2.50 en el plano de anteojos aéreos.

Este lente se fabrica con perfil asférico, está compuesto de acrílico hidrofóbico flexible con un índice de refracción de 1.55, cuenta con una asfericidad de -0.2 , posee un diseño apodizado biconvexo y en su centro hay siete anillos concéntricos. El diámetro de la región central es de $0.938 \mathrm{~mm}$, el diámetro del óptico es de $6 \mathrm{~mm}$, el diámetro total es de $13 \mathrm{~mm}$ y la estructura interna difractiva es de $3.4 \mathrm{~mm}$. La estructura externa refractiva es de $2.6 \mathrm{~mm}$, no tiene angulación en las hápticas y sus bordes son cuadrados?
El centro y la región periférica del lente son un 9.6 y $8.3 \%$ más largos, respectivamente, que su predecesor, el lente ReSTOR +3.0 , es decir, 0.938 frente a 0.856 para el centro, y 2.6 frente a 2.4 contra la periferia, respectivamente. Al ser implantado de manera bilateral, los pacientes consiguen alcanzar excelentes visiones (intermedia y de lejos) con un punto de visión de cerca.

Otro lente que también fabrica la misma casa comercial es el Acrysof IQ PanOptix (TFNT00). Este nuevo LIO multifocal y trifocal pretende no sacrificar la distancia de cerca, como lo hace el ReSTOR +2.50 , y tener un rango de visión mucho más amplio ${ }^{8}$.

El lente Acrysof IQ PanOptix está indicado para pacientes que tienen necesidad de trabajar a distancias intermedias y de cerca, específicamente a 0.4, 0.6 y $0.8 \mathrm{~m}$ de distancia. Tiene anillos concéntricos en la parte central de la óptica de $4.5 \mathrm{~mm}$ que le permiten un menor grado de pupilodependencia con respecto a otros lentes multifocales. El lente se diseñó para tener cuatro puntos focales: uno de cerca, dos intermedios y uno de lejos.

Este lente se fabrica con perfil asférico y está compuesto de acrílico hidrofóbico flexible con un índice de refracción de 1.55, al igual que la plataforma ya probada y conocida de Acrysof IQ. Cuenta con una asfericidad de -0.2 y en su centro hay 15 anillos concéntricos. El diámetro del óptico es de $6 \mathrm{~mm}$, el diámetro total es de $13 \mathrm{~mm}$, la estructura interna difractiva es de $4.5 \mathrm{~mm}$ y la estructura externa refractiva es de $1.5 \mathrm{~mm}$.

Tanto PanOptix como ReSTOR +2.50 están diseñados para ser insertados en la bolsa capsular en el momento de la facoemulsificación, y lo ideal para la cirugía es una capsulorrexis circular continua no superior a $5 \mathrm{~mm}$. Al ser implantado de manera bilateral, los pacientes llegan a alcanzar una excelente visión intermedia y de cerca sin sacrificar significativamente la visión de lejos ${ }^{9-14}$.

Los efectos adversos visuales secundarios al implante de lentes multifocales difractivos más frecuentes son: deslumbramientos, dificultad para la visión en 
condiciones de poca iluminación (sensibilidad al contraste disminuida) y halos, entre otros ${ }^{4,10,15}$. Sin embargo, al realizar pruebas de sensibilidad al contraste a los pacientes implantados con este lente se ha observado que no presentaban una disminución de la sensibilidad al contraste clínicamente significativa ${ }^{16}$.

Actualmente no existen estudios para conocer el pronóstico a largo plazo del implante bilateral ReSTOR +2.50 o Acrysof IQ PanOptix en pacientes mexicanos emétropes y sin comorbilidades. Además de esta situación, no se han comparado aún los efectos adversos que puede presentar el lente ReSTOR +2.50 frente al PanOptix en pacientes con catarata y presbicia, como en otros estudios ${ }^{10}$.

Se ha evaluado previamente el lente ReSTOR, con el que se han encontrado muy buenos resultados tanto de agudeza visual como de sensibilidad al contraste $9,10,13$.

Se han descrito ampliamente efectos adversos visuales, como halos y glare, sobre todo en condiciones mesópicas, aunque parece que éstos no repercuten significativamente en la calidad de vida de los pacientes; sin embargo, todo depende del tipo de actividad que éstos realicen ${ }^{8,10,17-19}$.

La calidad de vida de los pacientes siempre está directamente relacionada con la visión del paciente, motivo por el cual el Instituto Nacional de Ojos de EE.UU. desarrolló una encuesta validada tipo Likert de 25 ítems (VFQ-25-NEI) que mide la calidad de vida asociada a los problemas visuales. Esta encuesta determina diferentes componentes, entre ellos la satisfacción visual respecto a visión de cerca, visión a distancia, visión periférica, visión a color, función social en función de la visión e incluso actividades como conducir $^{20,21}$.

Existen diferentes métodos para evaluar la sensibilidad al contaste en los pacientes postoperados de un lente multifocal, entre los cuales se encuentra la tabla de sensibilidad al contraste Function Acuity Contrast Sensitivity Test, la cual se realiza en condiciones fotópicas a $85 \mathrm{~cd} / \mathrm{m}^{2}{ }^{16}$.

La implantación de LIO multifocales posteriores a cirugía de catarata solamente se realiza en pacientes sin alteraciones oftalmológicas aparentes, ya que se sabe que existe una notoria pérdida de la sensibilidad al contraste por el fenómeno de la difracción. Se ha presumido, sin embargo, que la pérdida no es significativa para el lente ReSTOR $+2.50^{22,23}$, aunque este fenómeno aún no se ha estudiado en profundidad en la población mexicana. Realizar este estudio también tiene la finalidad de conocer si implantar ReSTOR +2.50 disminuye 0 no la sensibilidad al contraste significativamente como para poder ofrecer la multifocalidad en pacientes a quienes se les contraindica esta tecnología.

La implantación de LIO con más de dos puntos focales, como es el caso del PanOptix, permite restablecer las visiones a diferentes distancias, lo que se traduce, en principio, en una mejor calidad de vida de los pacientes. Sin embargo, se sabe que, a mayor difracción de la luz a través de un medio como es el caso de este lente, se acentúan los efectos adversos visuales 8 ,10,17-19. El lente Acrysof IQ PanOptix ofrece una visión extendida en cuatro puntos focales que permite aprovechar la luz que pasa a través del óptico hasta en un $88 \%$, dejando así una pérdida de sensibilidad al contraste menor que otros lentes trifocales convencionales?

Es importante determinar si es eficaz un LIO con diferentes puntos focales y demostrar posibles ventajas o desventajas que tiene esta tecnología en comparación con otros lentes multifocales con tres o dos puntos focales.

Se pretende evaluar la cantidad/calidad de visión en los pacientes a quienes se les implanta de manera bilateral ReSTOR +2.50 y PanOptix, determinar si es significativa la pérdida de sensibilidad al contraste entre ambos lentes y el impacto que tienen tanto la visión como la calidad de ésta en la calidad de vida de la población mexicana.

Conocer con precisión el efecto que tiene sobre la población mexicana nos permite ofrecer a los pacientes las mejores opciones para presbicia en el contexto de cirugía de catarata.

\section{Objetivos generales}

Determinar datos objetivos y subjetivos de visión en los pacientes postoperados con ReSTOR +2.50 y PanOptix con catarata y presbicia, comparar los resultados y determinar si existe una diferencia clínica/estadística entre ellos.

\section{Material y métodos}

Se trata de un estudio observacional, longitudinal y prospectivo realizado en el Departamento de Segmento Anterior del Instituto de Oftalmología Fundación Conde de Valenciana I.A.P., en Ciudad de México. Se reclutaron de manera consecutiva pacientes candidatos para colocación de LIO multifocal, sin comorbilidades oculares, con astigmatismo $<1$ dioptría, sin antecedentes de cirugía ocular previa y que pudieran cumplir con el seguimiento a largo plazo. Fueron 
excluidos del estudio los pacientes a los que no se les pudo colocar de manera bilateral el mismo LIO o que tuvieran alteraciones que condicionaran el sesgo para los resultados obtenidos.

La evaluación preoperatoria se realiza de manera sistemática. Se ofrece LIO multifocal PanOptix o ReSTOR de manera aleatoria a los pacientes candidatos, y se les explican las ventajas y desventajas de cada LIO. La cirugía la llevan a cabo dos cirujanos pertenecientes al Departamento de Segmento Anterior del Instituto de Oftalmología Fundación Conde de Valenciana I.A.P. (E.C.M. y D.Z.C.), y la técnica utilizada fue stop and chop.

A todos los pacientes se les explicaron las ventajas y desventajas descritas por la casa comercial, y éstos escogieron su propio lente dependiendo de sus necesidades y actividades personales.

Se identificaron 14 ojos postoperados de facoemulsificación con implante ReSTOR +2.50 y 10 ojos postoperados de facoemulsificación con implante PanOptix en el Instituto de Oftalmología Fundación Conde de Valenciana I.A.P., los cuales fueron intervenidos durante el 1 de marzo al 31 de agosto de 2016.

Se citó directamente a los pacientes que fueron intervenidos en el periodo mencionado; proceso realizado a los seis meses cumplidos del postoperatorio en la totalidad de los pacientes.

Se evaluó la agudeza visual mejor corregida monocular en la totalidad de los pacientes con la cartilla de ETDRS; a 3 m y 60 y $33 \mathrm{~cm}$ con la cartilla de ETDRS para visión de lejos, intermedia y de cerca, respectivamente. Se realizó una curva de desenfoque monocular en la totalidad de los pacientes con la cartilla de ETDRS a $4 \mathrm{~m}$.

Se evaluaron los parámetros de sensibilidad al contraste mediante la cartilla de sensibilidad al contraste FACT, utilizando la refracción de la agudeza visual corregida del paciente y previa aplicación de lubricantes oculares.

La medición de la sensibilidad al contraste se realizó a una distancia de $3 \mathrm{~m}$ con respecto a la cartilla; la iluminación durante la prueba fue de $85 \mathrm{~cd} / \mathrm{m}^{2}$.

Se capturaron los valores de sensibilidad al contraste a diferentes frecuencias $(1.5,3,6,12$ y 18 ciclos por grado) y se transformó dicho valor a notación logarítmica para su mejor análisis estadístico (LogCS).

Se realizó la encuesta de calidad de vida VFQ-25 del Instituto Nacional de Ojos de EE.UU. (VFQ-25-NEI), con el formato único traducido al español de México (validado) al término de la revisión de los pacientes en ambos grupos.

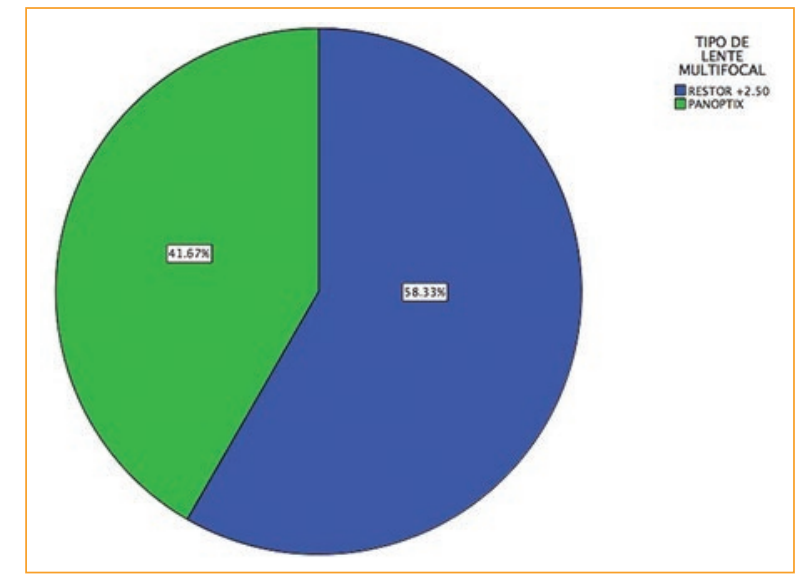

Figura 1. ReSTOR versus PanOptix: proporción de pacientes implantados durante el estudio.

A continuación, se realizó tonometría a los pacientes y se les aplicó un midriático con la finalidad de observar si existía opacidad de cápsula posterior o no, puesto que esto podía interferir en los resultados del estudio, en cuyo caso se cita en diferente momento para realizar capsulotomía con láser YAG y citar nuevamente al paciente a los 15 días para realizar nuevamente el estudio.

Inicialmente dividimos la población obtenida en dos grupos: el primero con ReSTOR +2.50 y el segundo con PanOptix. El análisis fue monocular.

Se realizaron pruebas de correlación (Spearman) entre las variables de sensibilidad al contraste, agudeza visual y el cuestionario validado VFQ-25, así como pruebas no paramétricas ( $U$ de Mann-Whitney) para comparar medias y determinar si existían diferencias estadísticamente significativas en las agudezas visuales y de sensibilidad al contraste, y el cuestionario VFQ-25.

Se utilizó el programa estadístico SPSS20.0, además del programa Excel Microsoft, para la captura de los datos.

\section{Resultados}

Se analizaron un total de 24 ojos de 12 pacientes. En la población se implantó un número equiparable de lentes en ambos ojos: ReSTOR (58.33\%) y PanOptix $(41.67 \%)$, como se muestra en la figura 1.

Se encontraron las siguientes agudezas visuales en ambos grupos a diferentes distancias de trabajo, como se muestra en la figura 2. Para el análisis de las gráficas conviene destacar que éste se realiza en 
Tabla 1. Comparación de resultados del cuestionario VFO-25 (resultado general y subescalas específicas)

\begin{tabular}{|c|c|c|c|c|c|c|c|c|}
\hline \multicolumn{9}{|l|}{ VF0-25-NEI } \\
\hline \multicolumn{2}{|c|}{ Tipo de lente multifocal } & \multirow{2}{*}{$\begin{array}{c}\text { Puntaje general } \\
\text { de VF0-25 } \\
96.4566\end{array}$} & \multirow{2}{*}{$\begin{array}{l}\text { Visión } \\
\text { general } \\
96.2500\end{array}$} & \multirow{2}{*}{$\begin{array}{c}\text { Actividades } \\
\text { de cerca } \\
88.5400\end{array}$} & \multirow{2}{*}{$\begin{array}{c}\text { Actividades } \\
\text { de lejos } \\
98.9575\end{array}$} & \multirow{2}{*}{$\begin{array}{l}\text { Conducir } \\
997.9000\end{array}$} & \multirow{2}{*}{$\begin{array}{c}\begin{array}{c}\text { Visión a } \\
\text { color }\end{array} \\
93.7500\end{array}$} & \multirow{2}{*}{$\begin{array}{c}\text { Visión } \\
\text { periférica } \\
100.00\end{array}$} \\
\hline ReSTOR + 2.50 & Media & & & & & & & \\
\hline & Mínimo & 90.30 & 85.00 & 62.50 & 95.83 & 91.60 & 75.00 & 100 \\
\hline & Máximo & 100.00 & 100.00 & 100.00 & 100.00 & 100.00 & 100.00 & 100 \\
\hline \multirow[t]{3}{*}{ Pan0ptix } & Media & 92.9790 & 90.0000 & 97.9150 & 93.7500 & & 100.0000 & 100.00 \\
\hline & Máximo & 88.63 & 80.00 & 91.66 & 75.00 & & 100.00 & 100 \\
\hline & Mínimo & 95.92 & 100.00 & 100.00 & 100.00 & & 100.00 & 100 \\
\hline
\end{tabular}

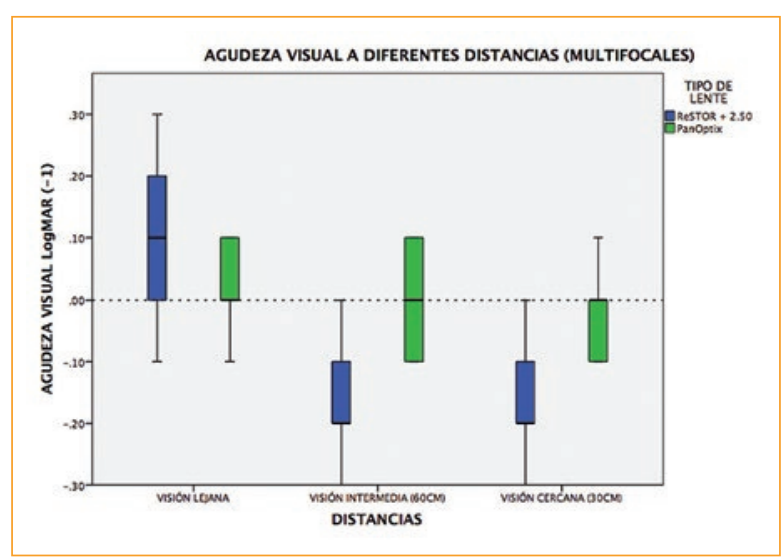

Figura 2. Agudeza visual a diferentes distancias de ambos LIO (lejana, intermedia y cercana).

unidades logarítmicas LogMAR y la gráfica se invierte $(-1)$ para un mejor análisis comparativo.

En esta última se muestra cómo la media de la agudeza visual de lejos en ambos grupos es notablemente buena; en distancias intermedia y de cerca, los valores son significativamente mejores en el grupo de PanOptix, a pesar de que la información comercial del ReSTOR favorece la visión intermedia en los pacientes. Se realiza prueba de Mann-Whitney, con la que se observan diferencias estadísticamente significativas para la visión intermedia y de cerca $(p<0.004$ y $p<0.002)$ (Fig. 3).

Se muestra la curva de desenfoque en ambos grupos. Se agregan los resultados de otro lente multifocal (Physiol/Finevision) en nuestra institución, cuya investigación se realiza de manera paralela $(n=25)$ (Fig. 4).
La curva de desenfoque del lente ReSTOR +2.50 se encuentra como lo esperado en la literatura médica, es decir, cae en la visión de cerca. Las otras dos gráficas que representan LIO con tres o más puntos focales siguen mostrando buenas agudezas visuales en un rango más amplio de visión (de $4 \mathrm{~m}$ a $3 \mathrm{~cm}$ ).

Los resultados de realizar la prueba de sensibilidad al contraste en ambos grupos se muestran en la figura 5 .

Marcamos los límites normales de la sensibilidad al contraste a diferentes frecuencias. Las líneas de color rojo y verde son medias de sensibilidad al contraste en diferentes frecuencias espaciales de ambos lentes, y se encuentran adyacentes al límite inferior normal, con lo que no existe una diferencia significativa al comparar ambos grupos en ninguna de las frecuencias $(p>0.315)$.

Ninguno de nuestros pacientes requirió capsulotomía por opacidad de la cápsula posterior durante el proceso de recolección de datos.

En la tabla 1 se muestran los valores del cuestionario VFQ-25 en diferentes LIO ReSTOR y PanOptix. Los valores resultan ser altos en la mayoría de las subescalas del VFQ-25. Al realizar la prueba comparativa para ambos LIO en las diferentes subescalas y valor general del VFQ-25, no se encontraron diferencias significativas $(p>0.341)$. No se pudieron comparar actividades como conducir, puesto que los pacientes del grupo de PanOptix no conducen. Excluir rubros como conducir no modifica el puntaje final del VFQ-25, pues el cuestionario lo permite.

Se realiza correlación entre las variables del VFQ, agudeza visual y sensibilidad al contraste, las cuales no arrojaron datos que pudieran ser valiosos para el estudio. No se encontró relación entre el VFQ y la agudeza visual o la sensibilidad al contraste. 


\begin{tabular}{|c|c|c|c|c|}
\hline \multicolumn{5}{|c|}{ Resumen de prueba de hipótesis } \\
\hline & Hipótes is nula & Test & Sig. & Decisión \\
\hline 1 & $\begin{array}{l}\text { La distribución de ACUDEZA } \\
\text { VISUAL LEJANA ( }>3 \mathrm{M} \text { ) es la } \\
\text { misma entre las categorias de } \\
\text { TIPO DE LENTE MULTIFOCAL. }\end{array}$ & $\begin{array}{l}\text { Prueba U de } \\
\text { Mann- } \\
\text { Whitney de } \\
\text { muestras } \\
\text { independiente } \\
\mathrm{s}\end{array}$ & $.165_{1}$ & $\begin{array}{l}\text { Retener la } \\
\text { hipótesis } \\
\text { nula. }\end{array}$ \\
\hline 2 & $\begin{array}{l}\text { La distribución de ACUDEZA } \\
\text { VISUAL INTERMEDIA (6O CMM es } \\
\text { la misma entre las Categorias de } \\
\text { TIPO DE LENTE MULTIFOCAL. }\end{array}$ & $\begin{array}{l}\text { Prueba U de } \\
\text { Mann- } \\
\text { Whitney de } \\
\text { muestras } \\
\text { independiente } \\
\mathrm{s}\end{array}$ & $.004_{1}$ & $\begin{array}{l}\text { Rechazar la } \\
\text { hipótesis } \\
\text { nula. }\end{array}$ \\
\hline 3 & $\begin{array}{l}\text { La distribución de ACUDEZA } \\
\text { VISUAL CERCANA ( } 30 C M \text { ) es la } \\
\text { misma entre las categorias de } \\
\text { TIPO DE LENTE MULTIFOCAL. }\end{array}$ & $\begin{array}{l}\text { Prueba U de } \\
\text { Mann- } \\
\text { Whitney de } \\
\text { muestras } \\
\text { independiente } \\
\mathrm{s}\end{array}$ & $.002_{1}$ & $\begin{array}{l}\text { Rechazar la } \\
\text { hipótesis } \\
\text { nula. }\end{array}$ \\
\hline & $\begin{array}{l}\text { muestran las significancias asin } \\
\text { e muestra la significancia exacta }\end{array}$ & $\begin{array}{l}\text { Sticas. El nivel } \\
\text { para esta prueb. }\end{array}$ & ignit & ancia es .05. \\
\hline
\end{tabular}

Figura 3. Resumen de la prueba de hipótesis. La visión resulta ser significativamente mejor en el lente Pan0ptix de acuerdo a la imagen previa.

\section{Discusión}

A día de hoy, los lentes ReSTOR +2.50 y PanOptix son una buena opción para restablecer la visión de cerca después de la cirugía de catarata, además de que siguen teniendo buena aceptación entre los cirujanos de segmento anterior.

Sabemos que el diseño propio de los LIO les permite tener diferentes distancias óptimas de visión. Mientras que el LIO ReSTOR +2.50 da preferencia a la visión intermedia y de lejos, el LIO PanOptix permite a los pacientes mayor independencia a los anteojos y recuperar buenas visiones desde los $4 \mathrm{~m}$ hasta los $30 \mathrm{~cm}$, como se mostró en la curva de desenfoque. En nuestro estudio sí se encontró una diferencia estadísticamente significativa de acuerdo a la prueba $U$ de Mann-Whitney a favor del LIO PanOptix para la visión intermedia y de cerca.

Evidentemente, habría que recalcar los efectos adversos que se han presentado con este tipo de lentes, como el glare, el deslumbramiento y los halos de luz alrededor de las luces. Estos efectos de primera instancia parecen estar más presentes en los pacientes a quienes se les implantó LIO PanOptix, y esta situación pudiera deberse a la cantidad de anillos y el tamaño de la zona difractiva que tiene este último lente ${ }^{24}$. Durante la realización del estudio, la totalidad de los pacientes implantados, tanto de ReSTOR como de PanOptix, comentaron fenómenos de glare/halos en el postoperatorio más inmediato, pero desaparecieron eventualmente. Este fenómeno no se pudo cuantificar objetivamente. Se realizó independientemente la medición de Point Spread Function con el equipo OPD III (Nidek) a los seis meses a todos los pacientes. Los resultados no mostraban indicios de presentar objetivamente halos en ninguno de los dos grupos o resultados como los que mostraron Gatinel, et al. ${ }^{24}$.

En cuanto a la satisfacción visual, ambos lentes estuvieron dentro de rangos aceptables si tomamos como premisa que la calificación más alta es de 100 dentro del puntaje de VFQ-25. Los valores presentados en nuestro trabajo son excelentes. Una vez realizada la comparación entre ambos lentes, no se encontró una diferencia estadísticamente significativa entre ambos, favoreciendo la teoría de que ambos lentes aportan buena calidad de vida y satisfacción visual. Se sabe de antemano que estos puntajes siguen con significancia clínica y estadística con respecto a los lentes monofocales ${ }^{25}$.

Es primordial destacar que hay que tomar en cuenta la ocupación de nuestros pacientes, además de las demandas visuales, puesto que de esto depende directamente su satisfacción visual. Sería preferible en los pacientes que aún tienen demandas laborales en computadora/oficina considerar PanOptix, puesto que estos lentes proporcionan un mayor rango de visión, y considerar los ReSTOR en aquellos pacientes en los que la demanda laboral depende directamente de la visión en condiciones de baja iluminación.

Se encontró que, al comparar valores de sensibilidad al contraste entre ambos lentes, no hubo una diferencia clínica o estadísticamente significativa entre ellos, motivo por el cual no debe ser un criterio para elegir entre un lente $u$ otro. Estos resultados obtenidos confirman la no colocación de LIO multifocales en casos en los que la sensibilidad al contraste esté disminuida.

Al realizar la comparación con el puntaje de VFQ-25, los puntajes en actividades tales como conducir (grupo ReSTOR) o discriminación de colores no son tan bajos como se esperaban, además de que el puntaje general del cuestionario VFQ-25 en ambos casos es equiparable entre sí.

La fortaleza de ambos lentes sigue siendo su diseño, los bordes cuadrados y sus hápticas, que les confieren un menor índice de opacidad de la cápsula posterior. Esto tiene ciertas ventajas, como una menor necesidad de aplicar láser YAG en los pacientes, y con ello menos complicaciones que disminuyan la visión a largo plazo. El diseño ha tenido mucha aceptación entre los cirujanos de catarata por la comodidad y seguridad de su implantación en la bolsa capsular (Fig. 6). 


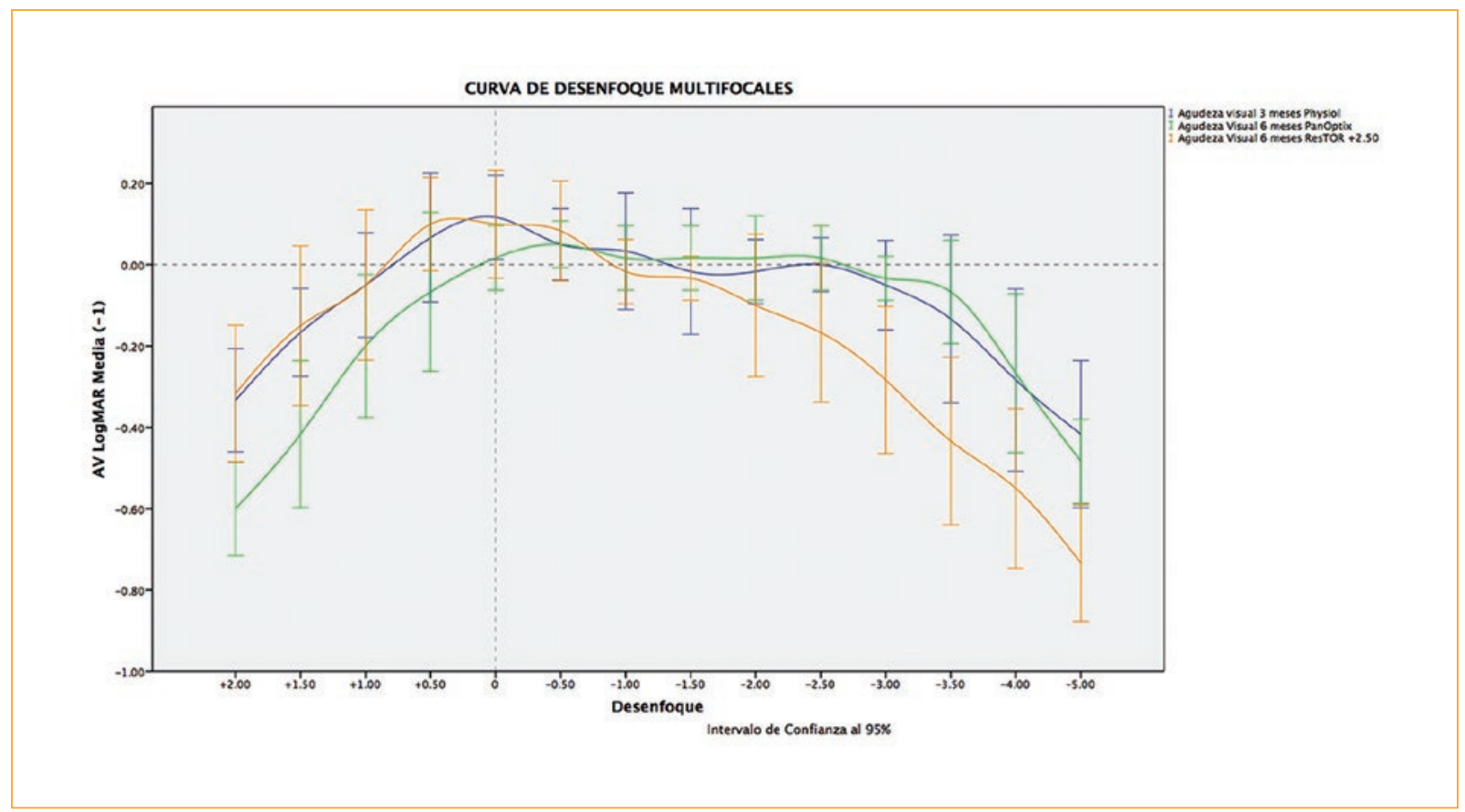

Figura 4. Curva de desenfoque. Se comparan tres LIO -Physiol (Fine vision), Pan0ptix (Alcon) y ReSTOR(+2.50)-, a los seis meses del postoperatorio. Los datos de la curva del Physiol fueron obtenidos de un estudio paralelo en el Instituto de Oftalmología Fundación Conde de Valenciana.

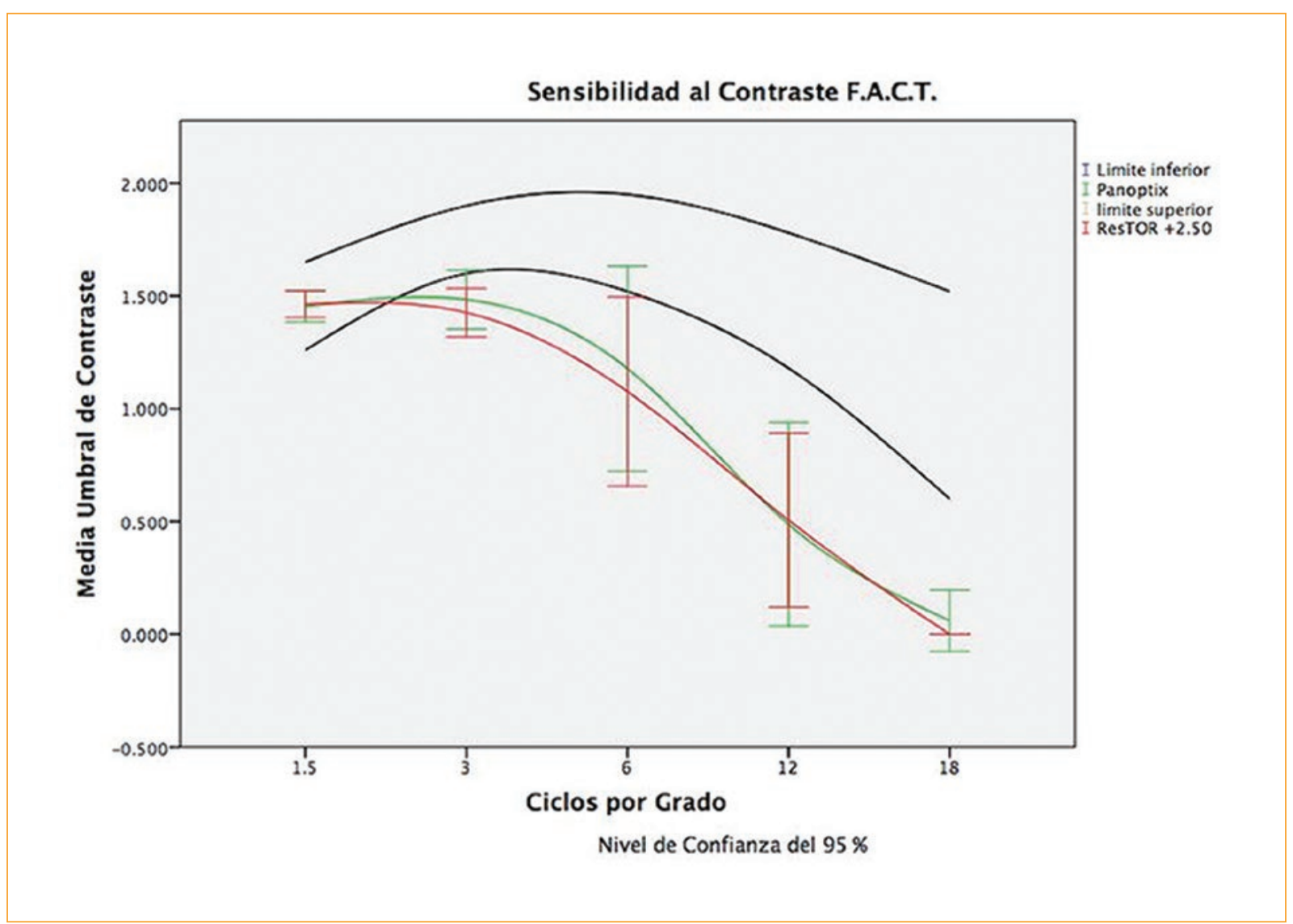

Figura 5. Prueba de sensibilidad al contraste en condiciones fotópicas. Se comparan PanOptix y Physiol. Las líneas negras delimitan el rango de la normalidad a través de las frecuencias. 
El estudio permitió determinar la sensibilidad al contraste en los pacientes postoperados con ReSTOR +2.50 en comparación con PanOptix. A pesar de una menor cantidad de anillos (ReSTOR), la sensibilidad al contraste es semejante, por lo que sigue sin recomendarse en los pacientes con comorbilidades que disminuyan la misma.

El estudio presenta ciertas debilidades, como no ser un estudio multicéntrico y el limitado tamaño de la muestra; sin embargo, nos puede aportar datos valiosos en el momento de elegir un lente multifocal.

Ambos lentes demostraron tener muy buena aceptación entre los pacientes, y parece ser que los efectos adversos como el glare y deslumbramiento están relacionados con el número de anillos que pueden o no desaparecer con la neuroadaptación de los pacientes.

\section{Conclusiones}

Al comparar ambos lentes se encontró una diferencia clínicamente y estadísticamente significativa cuando se trata de la visión de cerca o intermedia a favor de $\mathrm{Pa}$ nOptix. No hubo diferencias entre ambos lentes con respecto a la sensibilidad al contraste.

Las diferencias entre ambos lentes en cuanto al cuestionario validado de satisfacción visual VFQ-25NEI no fueron significativas. Los puntajes fueron altos para ambos lentes.

Esto último implica que, en el momento de decidir entre qué lente colocar al paciente, el criterio debe basarse principalmente en las necesidades de visión/actividades del paciente y sus posibilidades económicas.

Se ha especulado con respecto al fin o no de los LIO multifocales y bifocales como ReSTOR; sin embargo, parece que la respuesta depende directamente del valor en el mercado del mismo LIO.

Conocer las necesidades del paciente, así como su actividad, es de suma importancia para poder ofrecer el lente adecuado que corrija la presbicia después de una cirugía de catarata. A día de hoy ambos lentes siguen siendo una excelente opción para los pacientes, especialmente para aquéllos que laboralmente son inactivos y cuyas necesidades visuales no son tan altas en condiciones de penumbra.

\section{Financiamiento}

No se ha otorgado ningún financiamiento a la investigación y los autores declaran no tener ningún interés comercial en este protocolo.

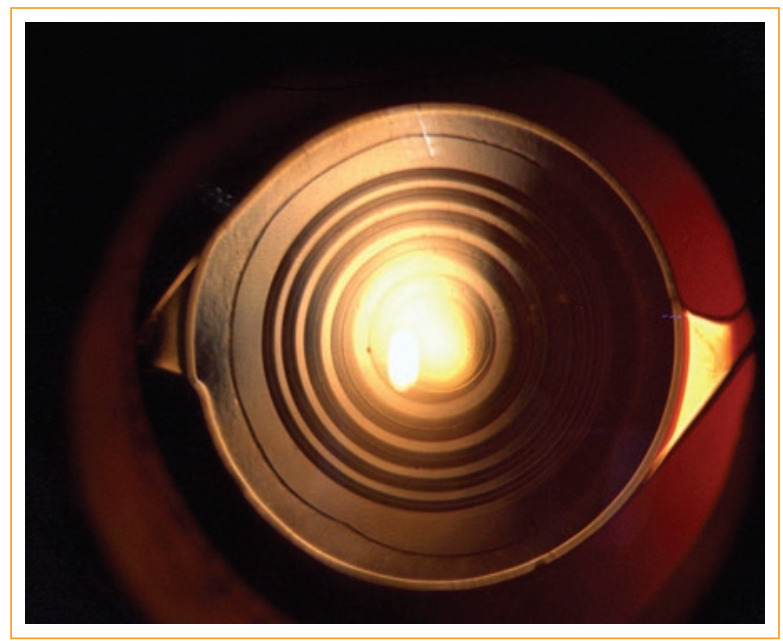

Figura 6. Lente Pan0ptix por retroiluminación.

\section{Conflicto de intereses}

No existe ningún conflicto de interés de los investigadores en este estudio.

\section{Responsabilidades éticas}

Protección de personas y animales. Los autores declaran que los procedimientos seguidos en el presente estudio se conformaron a las normas éticas del comité de experimentación humana responsable, de acuerdo con la Asociación Médica Mundial y la Declaración de Helsinki, y aprobado por los comités de Investigación y Ética en Investigación del Instituto de Oftalmología Fundación Conde de Valenciana I.A.P.

Confidencialidad de los datos. Los autores declaran que han seguido los protocolos de su centro de trabajo sobre la publicación de datos de pacientes.

Derecho a la privacidad y consentimiento informado. Los autores han obtenido el consentimiento informado de los pacientes y/o sujetos referidos en el artículo. Este documento obra en poder del autor de correspondencia.

\section{Bibliografía}

1. Dawson CR, Schwab IR. Epidemiology of cataract - a major cause of preventable blindness. Bull World Health Organ. 1981;59(4):493-501.

2. Auffarth GU, Dick HB. Multifocal intraocular lenses: a review. Ophthalmologe. 2001;98(2):127-7.

3. Bellucci R. Multifocal intraocular lenses. Curr Opin Ophthalmol. 2005:16(1):33-7.

4. Braga-Mele R, Chang D, Dewey S, et al. Multifocal intraocular lenses: relative indications and contraindications for implantation. J Cataract Refract Surg. 2014;40(2):313-22.

5. Davison JA, Simpson MJ. History and development of the apodized diffractive intraocular lens. J Cataract Refract Surg. 2006;32(5):849-58. 
6. Lehmann R, Waycaster C, Hileman K. A comparison of patient-reported outcomes from an apodized diffractive intraocular lens and a conventional monofocal intraocular lens. Curr Med Res Opin. 2006;22(12):2591-602.

7. Madrid-Costa D, Ruiz-Alcocer J, Ferrer-Blasco T, García-Lázaro S, Montes-Mico R. Optical quality differences between three multifocal intraocular lenses: bifocal low add, bifocal moderate add, and trifocal. J Refract Surg. 2013;29(11):749-54.

8. Lee S, Choi M, Xu Z, Zhao Z, Alexander E, Liu Y. Optical bench performance of a novel trifocal intraocular lens compared with a multifocal intraocular lens. Clin Ophthalmol. 2016;10:1031-8.

9. Gundersen KG, Potvin R. Comparative visual performance with monofocal and multifocal intraocular lenses. Clin Ophthalmol. 2013;7:1979-85.

10. Hayashi K, Ogawa S, Manabe SI, Hirata A. Visual outcomes in eyes with a distance-dominant diffractive multifocal intraocular lens with low near addition power. Br J Ophthalmol. 2015;99(11):1466-70.

11. Hutz WW, Eckhardt HB, Rohrig B, Grolmus R. Reading ability with 3 multifocal intraocular lens models. J Cataract Refract Surg. 2006;32(12):2015-21.

12. Hutz WW, Eckhardt HB, Rohrig B, Grolmus R. Intermediate vision and reading speed with array, Tecnis, and ReSTOR intraocular lenses. J Refract Surg. 2008;24(3):251-6.

13. Ji J, Huang X, Fan X, Luo M. Visual performance of Acrysof ReSTOR compared with a monofocal intraocular lens following implantation in cataract surgery. Exp Ther Med. 2013;5(1):277-81.

14. Kohnen $T$, Nuiits $R$, Levy $P$, Haefliger $E$, Alfonso JF. Visual function after bilateral implantation of apodized diffractive aspheric multifocal intraocular lenses with a +3.0 D addition. J Cataract Refract Surg. 2009; 35(12):2062-9.

15. Pieh $\mathrm{S}$, Weghaupt $\mathrm{H}$, Skorpik $\mathrm{C}$. Contrast sensitivity and glare disability with diffractive and refractive multifocal intraocular lenses. J Cataract Refract Surg. 1998;24(5):659-62.

16. Mayer S, Bohm T, Haberle H, Pham DT, Wirbelauer C. [Combined implantation of monofocal and multifocal intraocular lenses for presbyopia correction in cataract patients]. Klin Monbl Augenheilkd. 2008; 225(9):812-7.

17. Pedrotti E, Mastropasqua R, Passilongo M, Parisi G, Marchesoni I, Marchini G. Comparison of two multifocal intraocular lens designs that differ only in near add. J Refract Surg. 2014;30(11):754-60.

18. Maurino V, Allan BD, Rubin GS, Bunce C, Xing W, Findl O. Quality of Vision after Bilateral Multifocal Intraocular Lens Implantation: A Randomized Trial - AT LISA 809M versus AcrySof ReSTOR SN6AD1. Ophthalmology. 2015;122(4):700-10.

19. Carson D, Xu Z, Alexander E, Choi M, Zhao Z, Hong X. Optical bench performance of 3 trifocal intraocular lenses. J Cataract Refract Surg. 2016;42(9):1361-7.

20. Zhang F, Sugar A, Jacobsen G, Collins M. Visual function and spectacle independence after cataract surgery: bilateral diffractive multifocal intraocular lenses versus monovision pseudophakia. J Cataract Refract Surg. 2011;37(5):853-8.

21. Voskresenskaya A, Pozdeyeva N, Pashtaev N, Batkov Y, Treushnicov V, Cherednik V. Initial results of trifocal diffractive IOL implantation. Graefes Arch Clin Exp Ophthalmol. 2010;248(9):1299-306.

22. Castillo-Gómez A, Carmona-González D, Martínez-de-la-Casa JM, Palomino-Bautista C, García-Feijoo J. Evaluation of image quality after implantation of 2 diffractive multifocal intraocular lens models. J Cataract Refract Surg. 2009;35(7):1244-50.

23. Santhiago MR, Wilson SE, Netto MV, et al. Modulation transfer function and optical quality after bilateral implantation of $\mathrm{a}+3.00 \mathrm{D}$ versus $\mathrm{a}+4.00$ D multifocal intraocular lens. J Cataract Refract Surg. 2012;38(2):215-20.

24. Gatinel D, Loicq J. Clinically Relevant Optical Properties of Bifocal, Trifocal, and Extended Depth of Focus Intraocular Lenses. J Refract Surg. 2016;32(4):273-80.

25. Alió JL, Plaza-Puche AB, Piñero DP, Amparo F, Rodríguez-Prats JL, Ayala MJ. Quality of life evaluation after implantation of 2 multifocal intraocular lens models and a monofocal model. J Cataract Refract Surg. 2011;37(4):638-48. 